magnetic resonance. Detailed lifestyle information and anthropometric measurements were collected during childhood and adolescence. Metabolic parameters were measured multiple times per week for the first 9 weeks of life and again at follow-up visits.

Results Individuals that received IV lipids achieved significantly higher maximum cholesterol levels during the first 9 weeks of life than those that did not (mean $\pm \mathrm{SD}=4.38 \pm 1.65$ vs $3.12 \pm 0.78 \mathrm{mmol} /$ $1, p=0.006$ ). Dose given and number of days on IV lipids also associated with maximum cholesterol level during this period $(\mathrm{r}=0.557$, $\mathrm{p}<0.001$ and $\mathrm{r}=0.567, \mathrm{p}<0.001$, respectively). There was a graded relation between the maximum elevation in circulating cholesterol postnatally and aortic stiffness (aPWV) in young adulthood $(r=0.596, p<0.001)$. The greatest increase in stiffness was seen in the abdominal aorta, where distensibility was significantly reduced in the group that received IV lipids (mean $\pm S D=9.74 \pm 4.27$ vs $12.91 \pm 4.11 / \mathrm{mm} \mathrm{Hg} \times 10^{3}, \mathrm{p}=0.012$ ). There were no differences between the groups in other vascular or left ventricular measures. In a stepwise regression model, maximum cholesterol level achieved in the first few weeks of life was an independent predictor of aPWV in young adulthood $(\beta=0.596, p<0.001)$ and accounted for $30.9 \%$ of the variance in hierarchical multiple regression $(\beta=0.584, p<0.001)$. Conclusions Brief artificial elevation of cholesterol level in immediate postnatal life is associated with long term changes in aortic function independent of later cholesterol levels. The association is graded depending on the degree of elevation of circulating cholesterol. High cholesterol exposure during sensitive periods of early postnatal life may have long term impacts on the cardiovascular system.

\section{ETHNIC DIFFERENCES IN REPOLARISATION PATTERNS AND LEFT VENTRICULAR REMODELLING IN HIGHLY TRAINED MALE ADOLESCENT (14-18 YEARS) ATHLETES}

doi:10.1136/heartjnl-2011-300198.60

${ }^{1} \mathrm{~N}$ Sheikh, ${ }^{1} \mathrm{M}$ Papadakis, ${ }^{2} \mathrm{~F}$ Carre, ${ }^{2} \mathrm{G}$ Kervio, ${ }^{1} \mathrm{~J}$ Rawlins, ${ }^{3} \mathrm{~V}$ Panoulas, ${ }^{1} \mathrm{~N}$ Chandra, ${ }^{1} \mathrm{H}$ Raju, ${ }^{1} \mathrm{R}$ Bastiaenen, ${ }^{1} \mathrm{E}$ Behr, ${ }^{1} \mathrm{~S}$ Sharma. ${ }^{1}$ St. George's University of London, London, UK; ${ }^{2}$ French Institute of Health and Medical Research, University of Rennes, Rennes, France; ${ }^{3}$ University Hospital Lewisham, London, UK

Purpose Studies in adult, black athletes (BA) demonstrate a high prevalence of ECG repolarisation changes and echocardiographic left ventricular hypertrophy (LVH) that may overlap with hypertrophic cardiomyopathy (HCM). The prevalence of ECG repolarisation changes and echocardiographic LVH in adolescent BA, the group most vulnerable to exercise-related sudden death from HCM, is unknown. Methods This study evaluated 219 male adolescent BA (14-18 years, inclusive) with 12-lead ECG and 2-D echocardiography. Results were compared with 1440 male adolescent WA. Athletes with T wave inversions and morphological LVH were invited for further investigation with exercise stress test, $24 \mathrm{~h}$ Holter and CMR.

Results ST segment elevation was common in both groups but more frequent in BA $(63.5 \%$ vs $14.9 \%, \mathrm{p}<0.001)$, while ST segment depression was exceedingly rare. Both $\mathrm{T}$ wave inversions $(21.5 \%$ vs $2.9 \%, \mathrm{p}<0.001)$ and deep Twave inversions (11\% vs $0.3 \%, \mathrm{p}<0.001)$ were commoner in BA. Black athletes demonstrated greater left ventricular wall thickness $(10.4 \pm 1.6$ vs $9.4 \pm 1.2 \mathrm{~mm}, \mathrm{p}<0.001)$ compared to WA. Twenty-three (10.5\%) BA exhibited a left ventricular wall thickness $>12 \mathrm{~mm}$ vs only $6(0.4 \%)$ WA $(p<0.001)$. None of the athletes exhibited the broader phenotype of HCM on further investigation. In multivariable analysis black ethnicity was the strongest independent predictor for the presence of $\mathrm{T}$ wave inversions (OR 3.56, 95\% CI 1.56 to 8.13, $\mathrm{p}=0.003)$ and $\mathrm{LVH}(\mathrm{OR}$ $3.17,95 \%$ CI 1.77 to $5.71, \mathrm{p}<0.001)$.

Conclusions As with adult athletes, Twave inversions and LVH were more prevalent in adolescent BA compared to WA. These findings have important implications in the pre-participation screening era, particularly in countries with a high proportion of BA competing at elite level, since extrapolation of ECG and echocardiographic criteria, solely derived from Caucasian cohorts, would result in $25.6 \%$ of BA requiring further investigations for cardiac pathology.

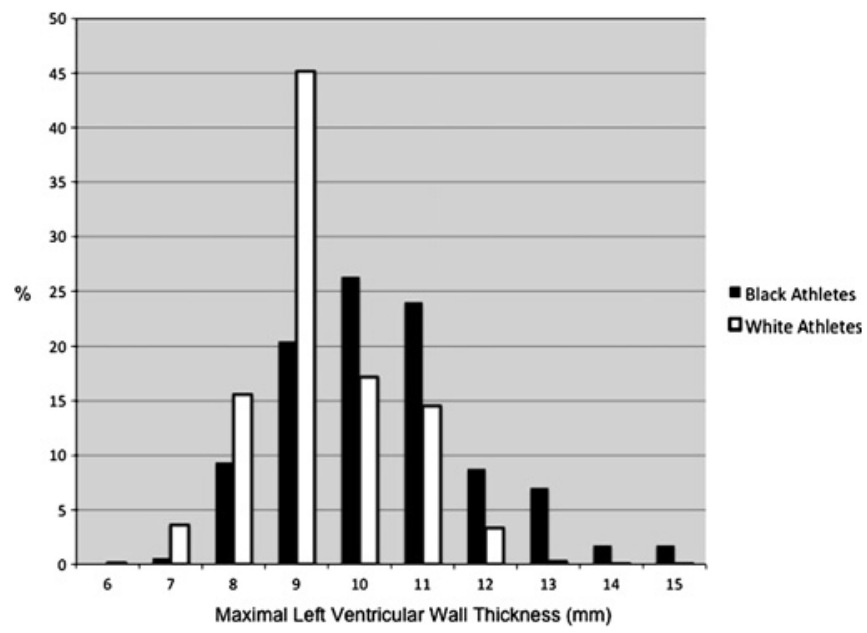

Abstract 60 Figure 1 Bar chart depicting the distribution of left ventricular wall thickness in black and white adolescent athletes.

\section{FIVE-MIN HEART RATE VARIABILITY CAN PREDICT OBSTRUCTIVE ANGIOGRAPHIC CORONARY DISEASE}

doi:10.1136/heartjnl-2011-300198.61

${ }^{1} \mathrm{D}$ Kotecha, ${ }^{2} \mathrm{G}$ New, ${ }^{1} \mathrm{M}$ Flather, ${ }^{3} \mathrm{D}$ Eccleston, ${ }^{3} \mathrm{H}$ Krum. ${ }^{1}$ Royal Brompton Hospital, London, UK; ${ }^{2}$ Box Hill Hospital, Melbourne, UK; ${ }^{3}$ Monash University, Melbourne, UK

Background Obstructive coronary artery disease (CAD) is evident in only half of patients referred for diagnostic coronary angiography. Five-minute heart rate variability (HRV) is a marker for autonomic control of the vasculature, which we hypothesised could be used to risk-stratify cardiac patients (the Alternative Risk Markers in Coronary Artery Disease (ARM-CAD) study.

Methods Resting HRV prior to elective coronary angiography was analysed in 470 participants with predominantly normal cardiac rhythm, regardless of comorbidity. The presence of obstructive CAD ( $\geq 50 \%$ stenosis) was regressed in a multivariate model including risk factors, ECG variables and medications.

Results Mean age was 65 years (SD 11), 67\% were male, 21\% had diabetes, mean blood pressure was $144 / 79 \mathrm{~mm} \mathrm{Hg}$ (SD 21/10) and $16 \%$ had impaired left-ventricular function. Patients with obstructive $\mathrm{CAD}$ had significantly reduced HRV, particularly in the low frequency (LF) range (median 180 vs $267 \mathrm{~ms}^{2}$ without CAD; $\mathrm{p}<0.001$ ). There was a linear trend according to the severity of CAD; the median LF power (IOR) in patients with normal coronaries was 275 (612), with minor coronary irregularities 255 (400),

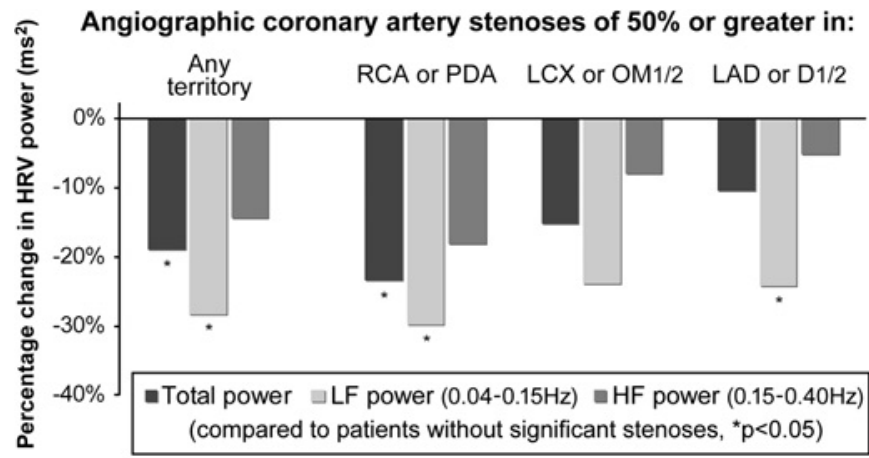

Abstract 61 Figure 1 